\title{
NONLINEAR SECOND ORDER ELLIPTIC PARTIAL DIFFERENTIAL EQUATIONS AT RESONANCE
}

\author{
R. IANNACCI, M. N. NKASHAMA, AND J. R. WARD, JR.
}

\begin{abstract}
In this paper we study the solvability of boundary value problems for semilinear second order elliptic partial differential equations of resonance type in which the nonlinear perturbation is not (necessarily) required to satisfy the Landesman-Lazer condition or the monotonicity assumption. The nonlinearity may be unbounded and some crossing of eigenvalues is allowed. Selfadjoint and nonselfadjoint resonance problems are considered.
\end{abstract}

\section{INTRODUCTION}

Let $\Omega \subseteq \mathbf{R}^{N}(N \geq 1)$ be a bounded domain with boundary $\partial \Omega$ of class $C^{1, \mu}, 0<\mu<1$, and let

$$
L u=\sum_{i=1}^{N} \sum_{j=1}^{N} \frac{\partial}{\partial x_{i}}\left(a_{i j}(x) \frac{\partial u}{\partial x_{j}}\right)-a_{0}(x) u
$$

and

$$
A u=\sum_{i=1}^{N} \sum_{j=1}^{N} \frac{\partial}{\partial x_{i}}\left(a_{i j}(x) \frac{\partial u}{\partial x_{j}}\right)+\sum_{i=1}^{N} b_{i}(x) \frac{\partial u}{\partial x_{i}}-a_{0}(x) u
$$

where

$$
\begin{gathered}
a_{i j}(x)=a_{j i}(x), 1 \leq i, j \leq N, a_{0}(x) \geq 0 \text { on } \Omega, \text { and } \\
\sum_{i=1}^{N} \sum_{j=1}^{N} a_{i j}(x) \xi_{i} \xi_{j}>0 \text { for all } x \in \bar{\Omega} \text { and all } \xi \in \mathbf{R}^{N} \backslash\{0\},
\end{gathered}
$$

( $\bar{\Omega}$ stands for the closure of $\Omega$ in $\mathbf{R}^{N}$ ). We assume that the coefficients of the differential operators $L$ and $A$ satisfy the following conditions

$$
\begin{gathered}
a_{i j} \in C^{1}(\bar{\Omega}), \quad 1 \leq i, j \leq N, \\
b_{i} \in L^{\infty}(\Omega), \quad 1 \leq i \leq N, \text { and } \\
a_{0} \in L^{\infty}(\Omega) .
\end{gathered}
$$

Received by the editors October 2, 1987 and, in revised form, July 28, 1988.

1980 Mathematics Subject Classification (1985 Revision). Primary 35J15, 35J25.

Key words and phrases. Boundary value problems, second order elliptic partial differential equations, (double) resonance, Leray-Schauder continuation method, topological degree. 
We shall consider the selfadjoint boundary value problems

$$
L u+\lambda_{1} u+g(x, u)=h \text { in } \Omega,\left.\quad u\right|_{\partial \Omega}=0,
$$

and the nonselfadjoint boundary value problems

$$
\cdot A u+\lambda_{1} u+g(x, u)=h \text { in } \Omega,\left.\quad u\right|_{\partial \Omega}=0
$$

where $\lambda_{1}$ is the first (resp. principal) eigenvalue of $-L$ (resp. $-A$ ), $h \in L^{p}(\Omega)$ with $p>N$, and $g: \Omega \times \mathbf{R} \rightarrow \mathbf{R}$ is a Carathéodory function which grows at most linearily i.e. $g(., u)$ is measurable for all $u \in \mathbf{R}, g(x,$.$) is continuous$ for a.e. $x \in \Omega$, and there exist a constant $c_{1}>0$ and a function $c_{2} \in L^{p}(\Omega)$, $p>N$, such that

$$
|g(x, u)| \leq c_{1}|u|+c_{2}(x)
$$

for a.e. $x \in \Omega$ and all $u \in \mathbf{R}$. Under the assumptions placed on $L$ and $A$ above, the Bony's maximum principle (see e.g. $[3,5]$ ) and the abstract KreinRutman theorem [23] imply the existence of a real simple eigenvalue $\lambda_{1}>0$ of the problem

$$
-L u=\lambda_{1} u \quad\left(\text { resp. }-A u=\lambda_{1} u\right),\left.\quad u\right|_{\partial \Omega}=0,
$$

of minimal modulus such that there is a corresponding smooth eigenfunction $\phi>0$ in $\Omega$ and $\partial \phi / \partial \eta<0$ on $\partial \Omega$, where $\partial / \partial \eta$ stands for the outward normal derivative. Moreover $\lambda_{1}$ is also an eigenvalue for the adjoint problem

$$
-A^{*} u=\lambda_{1} u,\left.\quad u\right|_{\partial \Omega}=0,
$$

such that there is a corresponding smooth eigenfunction $\phi^{*}>0$ in $\Omega$ and $\partial \phi^{*} / \partial \eta<0$ on $\partial \Omega[15]$.

In recent years much work has been devoted to the solvability of boundary value problems (1.1)-(1.2) in the nonresonance case. We refer to the papers by Berestycki and de Figueiredo [4], Dancer [10], de Figueiredo and Gossez [11], Mawhin [25] and the bibliography therein. When dealing with resonance problems, several results have been obtained by many authors when the nonlinearity $g(x, u)$ satisfies (among others) either a monotonicity assumption with respect to its second variable or a so called Landesman-Lazer condition

$$
\begin{aligned}
& \int g_{-}(x) \phi(x) d x<\int h(x) \phi(x) d x<\int g_{+}(x) \phi(x) d x \\
& \quad\left(\text { resp. } \int g_{-}(x) \phi^{*}(x) d x<\int h(x) \phi^{*}(x) d x<\int g_{+}(x) \phi^{*}(x) d x\right)
\end{aligned}
$$

where

$$
g_{-}(x)=\limsup _{u \rightarrow-\infty} g(x, u) \text { and } g_{+}(x)=\liminf _{u \rightarrow \infty} g(x, u) \text {. }
$$

The reader is referred to the papers by Ahmad [2], Berestycki and de Figueiredo [4], Brézis and Nirenberg [7], Dancer [10], Drabek [13], Fucik [14], Iannacci and Nkashama [19, 20], Mawhin, Ward and Willem [27, 28], Mawhin and Willem [29], Schechter, Shapiro and Snow [32]. 
On the other hand, very little is known on the solvability of boundary value problems (1.1)-(1.2) in the resonance case when the nonlinearity satisfies neither a monotoncity assumption nor a Landesman-Lazer condition (1.4). Let us mention here, for bounded nonlinearities, the papers by Cesari and Pucci [9], de Figueiredo and Ni [12], Gonçalves [16], Kannan, Nieto and Ray [22], Schaaf and Schmitt [31]; and, for unbounded nonlinearities in the case of ordinary differential equations, those ones by Gupta [18] and Iannacci and Nkashama [21].

It is the purpose of this paper to study the solvability of the second order partial differential equations (1.1)-(1.2), in which the nonlinearity is not necessarily required to satisfy monotonicity assumption or Landesman-Lazer condition. The nonlinearity may be unbounded and some crossing of eigenvalues is allowed. Our results are based on Leray-Schauder degree arguments and our proofs are somewhat a combination of ideas in Ahmad [2], Iannacci and Nkashama [21], Ward [32], together with some deep results on the maximum principle and regularity properties of second order elliptic partial differentail equations (see e.g. Amann and Crandall [3], Bony [5], Gilbarg and Trudinger [15] and Protter and Weinberger [30]).

This paper is organized as follows. In $\S 2$, we prove some preliminary results on piecewise linear problems

$$
L u+\lambda_{1} u+p_{+}(x) u^{+}-p_{-}(x) u^{-}=0,\left.\quad u\right|_{\partial \Omega}=0,
$$

and

$$
A u+\lambda_{1} u+p_{+}(x) u^{+}-p_{-}(x) u^{-}=0,\left.\quad u\right|_{\partial \Omega}=0,
$$

here $u^{+}(x)=\max (u(x), 0)$ and $u^{-}(x)=\max (-u(x), 0)$. In $\S \S 3$ and 4 , we state and prove our main results on the solvability of the selfadjoint and nonselfadjoint nonlinear boundary value problems $(1.1)-(1.2)$ (see Theorems 1-5).

Besides the classical real Lebesgue spaces $L^{p}(\Omega)$ and $C^{n}(\bar{\Omega})$ or $C^{n, \mu}(\bar{\Omega})$ of $n$-times continuously or Hölder continuously-differentiable real valued functions, we shall make use, in what follows, of the Sobolev spaces $H_{0}^{1}(\Omega)$ and $W^{2, p}(\Omega)$ (see e.g. Adams [1], Brézis [6] for definitions and properties).

For concluding this introduction, let us mention that when $\Omega \subseteq \mathbf{R}^{N}$ is a bounded domain whose boundary $\partial \Omega$ is a $C^{2}$-submanifold of dimension $N-1$ such that $\Omega$ lies locally on one side of $\partial \Omega$ and if $\partial \Omega$ is the disjoint union of two closed subsets $\Gamma_{0}$ and $\Gamma_{1}$ each of which is an $(N-1)$-dimensional submanifold of $\mathbf{R}^{N}$, then our results remain valid for second order elliptic partial differential equations with more general linear boundary condition $B u=$ 0 with

$$
B u=\left\{\begin{array}{ll}
u & \text { on } \Gamma_{0} \\
\frac{\partial u}{\partial \eta}+b_{0} u & \text { on } \Gamma_{1}
\end{array},\right.
$$


where $\eta \in C^{1}\left(\Gamma_{1} ; \mathbb{R}^{N}\right)$ is an outward pointing, nowhere tangent vector field on $\Gamma_{1}$, and $b_{0} \in C^{1}\left(\Gamma_{1} ; \mathbf{R}\right)$ satisfies $b_{0}(x) \geq 0$ on $\Gamma_{1}$. Thus $B$ is the Dirichlet boundary operator on $\Gamma_{0}$ and the Neumann or regular oblique derivative boundary operator on $\Gamma_{1}$ (either $\Gamma_{0}$ or $\Gamma_{1}$ may be empty). We restrict ourselves, to the case when $\partial \Omega$ is a $C^{1, \mu}$-submanifold of $\mathbb{R}^{N}$ with Dirichlet boundary operator on $\partial \Omega$, only for sake of bringing out the ideas involved clearly.

\section{PReliminary RESUltS ON PIECEWISE LINEAR PROBLEMS}

Throughout this paper, for a given linear operator $P$, we shall denote its nullspace (kernel) by $N(P)$. For each $u \in H_{0}^{1}(\Omega)$, let us write

$$
u(x)=\bar{u}(x)+\hat{u}(x)
$$

where $\bar{u}(x)=(1 / b)\left[\int u \phi d x\right] \phi(x), b=\int \phi^{2} d x$, and

$$
\hat{u}(x)=u(x)-\bar{u}(x) \text {, }
$$

so that, with obvious notations

$$
H_{0}^{1}(\Omega)=\bar{H}_{0}^{1}(\Omega) \oplus \widehat{H}_{0}^{1}(\Omega) .
$$

For $u \in L^{2}(\Omega)$, we define $u^{+}(x)=\max (u(x), 0)$ and $u^{-}(x)=\max (-u(x), 0)$, so that $u(x)=u^{+}(x)-u^{-}(x)$. Let $\lambda_{2} \in \mathbb{R}, \lambda_{2}>\lambda_{1}$, be the second eigenvalue of $-L$ with homogeneous Dirichlet boundary condition (see e.g. Gilbarg and Trudinger [15]) and let us set

$$
\alpha=\lambda_{2}-\lambda_{1}
$$

We have the following

Lemma 1. Let $\Gamma_{+}, \Gamma_{-} \in L^{p}(\Omega)$ be such that for a.e. $x \in \Omega$,

$$
0 \leq \Gamma_{ \pm}(x) \leq \alpha
$$

with

$$
\int_{u>0}\left[\alpha-\Gamma_{+}\right] w^{2} d x+\int_{u<0}\left[\alpha-\Gamma_{-}\right] w^{2} d x>0
$$

for all $w \in N\left(L+\lambda_{2} I\right), w \not \equiv 0$. Then, for all $p_{+}, p_{-} \in L^{p}(\Omega)$ with

$$
0 \leq p_{ \pm}(x) \leq \Gamma_{ \pm}(x)
$$

a.e. and all $u \in W^{2, p}(\Omega), p>N$, satisfying

$$
L u+\lambda_{1} u+p_{+}(x) u^{+}-p_{-}(x) u^{-}=0,\left.\quad u\right|_{\partial \Omega}=0,
$$

one has that $u \in N\left(L+\lambda_{1} I\right)$.

Proof. Let $p_{ \pm} \in L^{p}(\Omega)$ and $u \in W^{2, p}(\Omega)$ satisfy (2.3) and (2.4). Since $\Gamma_{+}$ and $\Gamma_{-}$fulfill the relation (2.2), it follows that

$$
\int_{u^{\prime}>0}\left[\alpha-p_{+}\right] w^{2} d x+\int_{u^{\prime}<0}\left[\alpha-p_{-}\right] w^{2} d x>0
$$


for all $w \in N\left(L+\lambda_{2} I\right), w \not \equiv 0$. Define $q: \Omega \times \mathbf{R} \rightarrow \mathbf{R}$ by

$$
q(x, u)= \begin{cases}p_{+}(x) & \text { if } x \in \Omega \text { and } u \geq 0, \\ p_{-}(x) & \text { if } x \in \Omega \text { and } u<0,\end{cases}
$$

so that

$$
0 \leq q(x, u) \leq \alpha
$$

for a.e. $x \in \Omega$ and all $u \in \mathbf{R}$, and for every $u \in L^{2}(\Omega)$ and a.e. $x \in \Omega$

$$
q(x, u(x)) u(x)=q(x, u) u^{+}-q(x, u) u^{-}=p_{+}(x) u^{+}(x)-p_{-}(x) u^{-}(x) .
$$

Now taking into account the orthogonality of $\bar{u}$ and $\hat{u}$ in $L^{2}(\Omega)$ and the fact that $(-L \hat{u}, \hat{u}) \geq \lambda_{2}(\hat{u}, \hat{u})$, one has

$$
\begin{aligned}
0 & =\int\left[L u+\lambda_{1} u+p_{+} u^{+}-p_{-} u^{-}\right][\bar{u}-\hat{u}] d x \\
& =\int\left[L u+\lambda_{1} u+q(x, u) u\right][\bar{u}-\hat{u}] d x \\
& =\int\left[(-L \hat{u}) \hat{u}-\left(\lambda_{1}+q(x, u)\right) \hat{u}^{2}\right] d x+\int q(x, u) \bar{u}^{2} d x \\
& \geq \int\left[(-L \hat{u}) \hat{u}-\lambda_{2} \hat{u}^{2}\right] d x \geq 0
\end{aligned}
$$

with equality if and only if $\hat{u}=w$ with $w \in N\left(L+\lambda_{2} I\right)$. In such a case, one has

$$
\int[\alpha-q(x, u(x))] w^{2} d x=0
$$

and

$$
\int q(x, u(x)) \bar{u}^{2} d x=0
$$

where $\bar{u} \in N\left(L+\lambda_{1} I\right), w \in N\left(L+\lambda_{2} I\right)$ and $u=\bar{u}+w$. Let

$$
\Omega_{v}=\{x \in \Omega: \bar{u}(x) \neq 0\} \text { and } \Omega_{w}=\{x \in \Omega: w(x) \neq 0\} .
$$

By equalities (2.6) and (2.7), one has necessarily

$$
\begin{array}{ll}
q(x, \bar{u}(x)+w(x))=0 & \text { for a.e. } x \in \Omega_{v}, \\
q(x, \bar{u}(x)+w(x))=\alpha & \text { for a.e. } x \in \Omega_{w},
\end{array}
$$

and hence the (Lebesgue) measure of the set $\boldsymbol{\Omega}_{v} \cap \boldsymbol{\Omega}_{w}$ is zero. If $\boldsymbol{\Omega}_{v}=\varnothing$, then $u=w$ and the equality (2.6) becomes

$$
0=\int[\alpha-q(x, w(x))] w^{2} d x=\int_{w>0}\left[\alpha-p_{+}\right] w^{2} d x+\int_{w<0}\left[\alpha-p_{-}\right] w^{2} d x,
$$

so that, by relation (2.5), $w=0$ and hence $u=0$. If $\Omega_{v} \neq \varnothing$, it follows, from the properties of the eigenfunction $\phi$ and the fact that $\bar{u}(x)=a \phi(x)$ with $a \in \mathbf{R}$, that $\Omega_{v}=\Omega$. Therefore, one must have $q(x, u(x))=0$ for a.e. $x \in \Omega$. By equality (2.6), one gets $w=0$ and hence $u=\bar{u}$. Thus, in both cases, $u=\bar{u} \in N\left(L+\lambda_{1} I\right)$ and the proof is complete. 
While the preceding lemma will allow us to get an existence result for (1.1) when the nonlinearity "lies" between the two first eigenvalues, the following lemma will allow us to derive existence results for (1.1) when the nonlinearity "jumps over" the second eigenvalue. In fact, we can cross any number of higher order eigenvalues.

Lemma 2. Let $\Gamma_{-} \in L^{p}(\Omega), p>N$, be a function such that $\Gamma_{-}(x) \geq 0$ for a.e. $x \in \Omega$. Then there exists a constant $d=d\left(\Gamma_{-}\right)>0$ such that for all $p_{+}, p_{-} \in L^{p}(\Omega)$ with

$$
0 \leq p_{+}(x) \leq d, \quad 0 \leq p_{-}(x) \leq \Gamma_{-}(x)
$$

for a.e. $x \in \Omega$, and all $u \in W^{2, p}(\Omega), p>N$, verifying equation (2.4), one has that only one of the following assertions holds true:

(i) $u=0$ on $\Omega$;

(ii) $u(x)>0$ for all $x \in \Omega$ and $\partial u / \partial \eta<0$ on $\partial \Omega$;

(iii) $u(x)<0$ for all $x \in \Omega$ and $\partial u / \partial \eta>0$ on $\partial \Omega$.

Proof. If $u(x) \neq 0$ for some $x \in \Omega$, then either (ii) or (iii) holds true.

Let us assume that the conclusion of the lemma does not hold. Then, for each $n \in \mathbb{N}$, there exist $p_{ \pm}^{n} \in L^{p}(\Omega)$ and $u_{n} \in W^{2, p}(\Omega), p>N$, with

$$
\begin{gathered}
0 \leq p_{+}^{n}(x) \leq 1 / n \text { a.e. on } \Omega, \\
0 \leq p_{-}^{n}(x) \leq \Gamma_{-}(x) \text { a.e. on } \Omega, \\
\left|u_{n}\right|_{C^{1, \mu}}=1
\end{gathered}
$$

such that

$$
L u_{n}+\lambda_{1} u_{n}+p_{+}^{n}(x) u_{n}^{+}-p_{-}^{n}(x) u_{n}^{-}=0,\left.\quad u_{n}\right|_{\partial \Omega}=0,
$$

and neither (ii) nor (iii) is fulfilled.

Since $W^{2, p}(\Omega)$ and $C^{1, \mu}(\bar{\Omega})$ are compactly embedded into $C^{1}(\bar{\Omega})$ ([1]), by using the weak closedness of $L$ [2, pp. 150-151], relations (2.9), the standard $L^{p}$-estimate $|u|_{W^{2, p}} \leq c|L u|_{L^{p}}$ for second order elliptic partial differential equations [15], and the fact that $W^{2, p}(\Omega)$ is a reflexive Banach space [6], we can assume (by going if necessary to a subsequence) that there exist $u \in W^{2, p}(\Omega)$, $p_{-} \in L^{p}(\Omega)$, with

$$
|u|_{C^{1, \mu}}=1, \quad 0 \leq p_{-}(x) \leq \Gamma_{-}(x) \text { a.e. on } \Omega \text {, }
$$

such that

$$
\begin{gathered}
u_{n}-u \text { in } W^{2, p}(\Omega), \quad u_{n} \rightarrow u \text { in } C^{1, \mu}(\bar{\Omega}), \\
p_{-}^{n} \rightarrow p_{-} \text {in } L^{p}(\Omega), \quad p_{+}^{n} \rightarrow 0 \text { in } L^{\infty}(\Omega), \quad \text { as } n \rightarrow \infty,
\end{gathered}
$$

and

$$
L u+\lambda_{1} u-p_{-}(x) u^{-}=0,\left.\quad u\right|_{\partial \Omega}=0 .
$$


By taking the inner product (in $L^{2}(\Omega)$ ) of (2.13) with the eigenfunction $\phi$, we deduce that $p_{-}(x)=0$ (a.e.) whenever $u(x)<0$. Hence, $(2.13)$ reduces to

$$
L u+\lambda_{1} u=0,\left.\quad u\right|_{\partial \Omega}=0, \quad u \not \equiv 0 .
$$

Therefore, by the properties of $N\left(L+\lambda_{1} I\right) \backslash\{0\}$, either $u(x)>0$ for all $x \in \Omega$ and $(\partial u / \partial \eta)(x)<0$ for all $x \in \partial \Omega$ or $u(x)<0$ for all $x \in \Omega$ and $(\partial u / \partial \eta)(x)>0$ for all $x \in \partial \Omega$. Since $u_{n} \rightarrow u$ in $C^{1, \mu}(\bar{\Omega})$, this implies that there exists a non-negative integer $n_{0}$ such that for each $n \geq n_{0}$ either $u_{n}(x)>0$ for all $x \in \Omega$ and $\left(\partial u_{n} / \partial \eta\right)(x)<0$ for all $x \in \partial \Omega$ or $u_{n}(x)<0$ for all $x \in \Omega$ and $\left(\partial u_{n} / \partial \eta\right)(x)>0$ for all $x \in \partial \Omega$. We get a contradiction to the fact that for each $n \in \mathbb{N}, u_{n}$ does satisfy neither assertion (ii) nor (iii) of the lemma and the proof is complete.

Remark 1. If $\Omega \subseteq \mathbb{R}^{N}$ is a bounded domain whose boundary $\partial \Omega$ is a $C^{2}$ submanifold of dimension $N-1$ such that $\Omega$ lies locally on one side of $\partial \Omega$, the above lemmas may be proved for Newton (regular oblique derivative) boundary value problems as mentioned in the introduction. The $L^{p}$-estimate to be used, in that case, is given by Proposition 2 in Amann and Crandall [3, p. 783]. Note that, for Neumann boundary conditions, the statements and proofs of the above lemmas may be significantly simplified. In the case of Dirichlet boundary conditions, the underlying principle in Lemma 2 (though not in the same generality given here) also was observed by Dancer [10].

On the other hand, if $\Gamma_{-}$is a constant in Lemma 2, then by using the "shooting method" in one dimensional case $(N=1)$, one can find the explicit relationship between $\Gamma_{-}$and $d$ (see e.g. Drabek [13], Dancer [10] and references therein).

Results similar to those proved above hold true for nonselfadjoint piecewise linear problems. We shall state two results. The first is due to Ahmad [2, pp. 149-152], while the second one is similar to Lemma 2 herein.

Lemma 3 [2]. There exists a constant $d^{*}>0$ such that for all $p_{ \pm} \in L^{p}(\Omega)$ with

$$
0 \leq p_{ \pm}(x) \leq d^{*} \text { a.e. on } \Omega \text {, }
$$

and all $u \in W^{2, p}(\Omega), p>N$, satisfying the equation

$$
A u+\lambda_{1} u+p_{+}(x) u^{+}-p_{-}(x) u^{-}=0,\left.\quad u\right|_{\partial \Omega}=0,
$$

one has that the conclusion of Lemma 2 holds true.

Note that the approach used in the proof of Lemma 2 herein gives a simpler proof of Lemma 3. For selfadjoint second order uniformly strongly elliptic operators, it is known that $d^{*}<\alpha \quad\left(=\lambda_{2}-\lambda_{1}\right)$; in that case, we have got much more precise results (see Lemmas 1 and 2 herein). On the other hand, for nonselfadjoint elliptic operators considered herein, by assuming that the equation

$$
A u+\lambda_{1} u+d u=0,\left.\quad u\right|_{\partial \Omega}=0, \quad \text { with } d>0,
$$


has a nontrivial solution, it is easy to see that $d \geq \alpha$, where now $\alpha>0$ is the largest number such that

$$
\left(A u+\lambda_{1} u, u\right) \geq(-1 / \alpha)\left|A u+\lambda_{1} u\right|_{L^{2}}^{2}
$$

for all $u \in H^{2}(\Omega) \cap H_{0}^{1}(\Omega)$. We are ensured that Lemma 3 holds for any $d^{*}<\alpha$. For the existence of such a number $\alpha$, we refer to Berestycki and de Figueiredo [4] or Brézis and Nirenberg [7].

Lemma 4. Let $\Gamma_{-} \in L^{p}(\Omega), p>N$, be a nonnegative function a.e. on $\Omega$. Then there exists a constant $d=d\left(\Gamma_{-}\right)>0$ such that for all $p_{ \pm} \in L^{p}(\Omega)$ satisfying

$$
\begin{gathered}
0 \leq p_{+}(x) \leq d, \\
0 \leq p_{-}(x) \leq \Gamma_{-}(x)
\end{gathered}
$$

for a.e. $x \in \Omega$, and all $u \in W^{2, p}(\Omega), p>N$, solving (2.15), one has that the conclusion of Lemma 2 holds, true. (Note that there is no restriction on the upper bound for $\Gamma_{-}$).

Proof. It parallels the proof of Lemma 2, in which one replaces the operator $L$ by $A$, up to the point (2.13). Now, taking the inner product (in $L^{2}(\Omega)$ ) of (2.13) (where $L$ is replaced by $A$ ) with the eigenfunction $\phi^{*}$, we deduce that $p_{-}(x)=0$ (a.e.) whenever $u(x)<0$. Therefore (2.13) reduces to $A u+\lambda_{1} u=$ $0,\left.u\right|_{\partial \Omega}=0$. One can reach a contradiction as in the proof of Lemma 2 and the proof is complete.

\section{SELFADJOINT SEMILINEAR RESONANCE PROBLEMS}

Let $g: \Omega \times \mathbf{R} \rightarrow \mathbf{R}$ be a Carathéodory function which grows at most linearily (see $\S 1$ and condition (1.3)). We assume, without loss of generality, that the following upper bounds are nonnegative

$$
\begin{aligned}
& \lim _{u \rightarrow \infty} \sup g(x, u) / u \leq \Gamma_{+}(x), \quad \text { a.e. on } \Omega \\
& \lim _{u \rightarrow-\infty} \sup g(x, u) / u \leq \Gamma_{-}(x), \quad \text { a.e. on } \Omega .
\end{aligned}
$$

Obviously, according to the growth condition (1.3), we can suppose, without loss of generality, that $\Gamma_{ \pm} \in L^{p}(\Omega), p>N$.

Theorem 1. Let us assume that

$$
g(x, u) u \geq 0
$$

for a.e. $x \in \Omega$ and all $u \in \mathbf{R}$. Moreover, suppose that the functions $\Gamma_{+}, \Gamma_{-}$, given in relation (3.1), are such that

$$
0 \leq \Gamma_{ \pm}(x) \leq \alpha\left(=\lambda_{2}-\lambda_{1}\right) \text { for a.e. } x \in \Omega,
$$

with

$$
\int_{u>0}\left[\alpha-\Gamma_{+}\right] w^{2} d x+\int_{u<0}\left[\alpha-\Gamma_{-}\right] w^{2} d x>0
$$


for all $w \in N\left(L+\lambda_{2} I\right), w \not \equiv 0$. Then equation (1.1) has at least one solution $u \in W^{2, p}(\Omega) \cap H_{0}^{1}(\Omega)$ for any $h \in L^{p}(\Omega), p>N$, with

$$
\int_{\Omega} h \phi d x=0 \text {. }
$$

moreover $u \in C^{1, \mu}(\bar{\Omega})$. (Recall that $\phi$ is the unique eigenfunction associated to the eigenvalue $\lambda_{1}$ of $L$.)

Proof. For some fixed constant $\gamma$ with $0<\gamma<\alpha$, let the operator

$$
E: W^{2, p}(\Omega) \cap H_{0}^{1}(\Omega) \subseteq C^{1}(\bar{\Omega}) \rightarrow L^{p}(\Omega)
$$

be defined by

$$
E u=L u+\lambda_{1} u+\gamma u .
$$

To prove that (1.1) has at least one solution, it suffices, according to the LeraySchauder continuation method (see [24]), and the compact embedding of $W^{2, p}(\Omega), p>N$, into $C^{1}(\bar{\Omega})$ (see [1]), to show that there exists a constant $\rho>0$ such that

$$
|u|_{C^{1}}<\rho
$$

for all possible solutions $u \in W^{2, p}(\Omega) \cap H_{0}^{1}(\Omega)$ of the homotopy

$$
L u+\lambda_{1} u+(1-t) \gamma u+\operatorname{tg}(x, u)=\operatorname{th}(x),
$$

$t \in[0,1)$, (with $\rho$ independent of $u$ and $t$ ). It is clear that for $t=0$, (3.7) has only the trivial solution. Now, let us assume that the relation (3.6) does not hold true, then there exist sequences $\left(t_{n}\right)$ in the open interval $(0,1)$ and $\left(u_{n}\right)$ in $W^{2, p}(\Omega)$ with $\left|u_{n}\right|_{C^{1}} \geq n$ for all $n \in \mathrm{N}$ such that

$$
E u_{n}=t_{n}\left[\gamma u_{n}-g\left(x, u_{n}\right)+h(x)\right],\left.\quad u_{n}\right|_{\partial \Omega}=0 .
$$

Setting $v_{n}=\left(u_{n} /\left|u_{n}\right|_{C^{1}}\right)$, we have

$$
E v_{n}=t_{n}\left[\gamma v_{n}-\left(g\left(x, u_{n}\right) /\left|u_{n}\right|_{C^{1}}\right)+\left(h /\left|u_{n}\right|_{C^{1}}\right)\right],\left.\quad v_{n}\right|_{\partial \Omega}=0 .
$$

By the growth condition (1.3), it follows that $\left(g\left(x, u_{n}\right) /\left|u_{n}\right|_{C^{1}}\right)$ is bounded in $L^{p}(\Omega)$, so that the right-hand member of equality (3.9) is bounded in $L^{p}(\Omega)$. By using $L^{p}$-estimate, and the compact embedding of $W^{2, p}(\Omega)$ into $C^{1}(\bar{\Omega})$, we deduce from (3.9), (going if necessary to a subsequence relabeled $\left(v_{n}\right)$ ), that there exist $v \in C^{1}(\bar{\Omega}), t \in[0,1]$, such that

$$
\begin{gathered}
v_{n} \rightarrow v \quad \text { in } C^{1}(\bar{\Omega}), \quad t_{n} \rightarrow t \text { as } n \rightarrow \infty, \\
|v|_{C^{1}}=1 \text { and }\left.v\right|_{\partial \Omega}=0 .
\end{gathered}
$$

On the other hand, by (3.9), we get that $\left|L v_{n}\right|_{L^{p}} \leq c$ for some constant $c>0$. Therefore, by using the fact that $L^{p}(\Omega)$ is a reflexive Banach space and the weak closedness of the operator $L$, we get that $v \in W^{2, p}(\Omega) \cap H_{0}^{1}(\Omega), L v_{n}-L v$ in $L^{p}(\Omega)$ and $v$ solves the equation

$$
E v=t[\gamma v-K(x)],\left.\quad v\right|_{\partial \Omega}=0,
$$


where $K(x)$ is the weak limit in $L^{p}(\Omega)$ of the sequence $\left(g\left(x, u_{n}\right) /\left|u_{n}\right|_{C^{1}}\right)$. Let us mention, here, that (3.11) implies that $t \neq 0$. Indeed, if $t=0$, then by using the definition of the operator $E$, we deduce, from (3.11), that $v=0$, which would contradict the fact that $|v|_{C^{1}}=1$. In order to bring out all properties of the function $v$, we need to analyze a little bit carefully the function $K(x)$ in (3.11). Let us denote by $k_{v}(x)$ the function defined by $K(x) / v(x)$ if $v(x) \neq 0$ and $k_{v}(x)=0$ if $v(x)=0$. It turns out that

$$
\begin{array}{ll}
0 \leq k_{v}(x) \leq \Gamma_{+}(x) & \text { if } v(x)>0, \\
0 \leq k_{v}(x) \leq \Gamma_{-}(x) & \text { if } v(x)<0,
\end{array}
$$

we refer to [4, pp. 108-110]. Therefore (3.11) is equivalent to

$$
E v-t\left[\gamma v-k_{v}(x) v\right]=0,\left.\quad v\right|_{\partial \Omega}=0 .
$$

By setting $k_{v}^{+}(x)=k_{v}(x)$ for $v(x)>0$, and $k_{v}^{-}(x)=k_{v}(x)$ for $v(x)<0$ and observing that

$$
k_{v}(x) v(x)=k_{v}^{+}(x) v^{+}(x)-k_{v}^{-}(x) v^{-}(x),
$$

we obtain that $(3.13)$ is equivalent to

$$
\begin{aligned}
& L v+\lambda_{1} v+\left[(1-t) \gamma+t k_{v}^{+}(x)\right] v^{+} \\
& -\left[(1-t) \gamma+t k_{v}^{-}(x)\right] v^{-}=0,\left.\quad v\right|_{\partial \Omega}=0 .
\end{aligned}
$$

Hence, by inequalities (3.12), and Lemma 1 in which

$$
\begin{aligned}
& p_{+}(x)=(1-t) \gamma+t k_{v}^{+}(x), \\
& p_{-}(x)=(1-t) \gamma+t k_{v}^{-}(x),
\end{aligned}
$$

we deduce that $v \in N\left(L+\lambda_{1} I\right) \backslash\{0\}$, so that we have either $v>0$ in $\Omega$ and $\partial v / \partial \eta<0$ on $\partial \Omega$, or $v<0$ in $\Omega$ and $\partial v / \partial \eta>0$ on $\partial \Omega$. Let us assume that $v>0$ and finish the proof (the proof for the case $v<0$ is similar).

Since, by (3.10), $v_{n} \rightarrow v$ in $C^{1}(\bar{\Omega})$ with $v(x)>0$ in $\Omega$ and $\partial v / \partial \eta<0$ on $\partial \Omega$, we get that there exists $n_{0} \in \mathrm{N}$ such that for $n>n_{0}, v_{n}(x)>0$ for all $x \in \Omega$. Therefore, for all $n>n_{0}$

$$
u_{n}(x)>0 \text { for all } x \in \Omega \text {, }
$$

where $u_{n}$ is a solution of the homotopy (3.8) which is now equivalent to

$$
\begin{gathered}
L u_{n}+\lambda_{1} u_{n}+\left(1-t_{n}\right) \gamma u_{n}+t_{n} g\left(t, u_{n}\right)=t_{n} h(x), \\
\left.u_{n}\right|_{\partial \Omega}=0, \quad \text { for } n>n_{0} .
\end{gathered}
$$

Now taking the inner product (in $L^{2}(\Omega)$ ) of (3.17) with the eigenfunction $\phi$, observing that $t_{n} \in(0,1)$, and taking into account assumption (3.5), we deduce that, for each $n>n_{0}$,

$$
\int_{\Omega} g\left(x, u_{n}(x)\right) \phi(x) d x<0 .
$$


This is a contradiction since, by (3.16) and assumption (3.2), we have

$$
g\left(x, u_{n}(x)\right) \phi(x) \geq 0 \text { a.e. in } \Omega
$$

for each $n>n_{0}$ and the proof is complete.

Remark 2. Theorem 1 generalizes main results in de Figueiredo and Ni [12], Gonçalves [16], Gupta [17, 18], Iannacci and Nkashama [21], and Ward [33].

It is obvious that in Theorem 1, the function $f(x, u)=\lambda_{1} u+g(x, u)$ "lies" between the two first eigenvalues. We shall, now, present a result on crossing of eigenvalues. Actually either $\lim \sup _{u \rightarrow \infty} g(x, u) / u$ or $\lim _{\sup _{u \rightarrow-\infty}} g(x, u) / u$ can cross any number of eigenvalues.

Theorem 2. Let us assume that inequality (3.2) holds true. Let $\Gamma_{-}(x) \geq c>$ $0, c \in \mathbf{R}$, a.e. on $\Omega, \Gamma_{-}$given in relation (3.1), and let $d=d\left(\Gamma_{-}\right)>0$ be a constant associated to $\Gamma_{-}$by Lemma 2 . Moreover suppose that the function $\Gamma_{+}$, given in relation (3.1), is such that

$$
0 \leq \Gamma_{+}(x) \leq d
$$

for a.e. $x \in \Omega$. Then (1.1) has at least one solution for any $h \in L^{p}(\Omega), p>N$, satisfying the orthogonality condition (3.5). Moreover that solution belongs to $C^{1, \mu}(\bar{\Omega})$. One can obtain a similar result when the roles of $\Gamma_{+}$and $\Gamma_{-}$are interchanged.

Proof. It parallels the proof of Theorem 1. Let the constant $d_{1}$ be defined by

$$
d_{1}=\min \left\{d\left(\Gamma_{-}\right), c, \alpha / 2\right\} .
$$

We consider the following homotopy

$$
L u+\lambda_{1} u+(1-t) d_{1} u+\operatorname{tg}(x, u)=\operatorname{th}(x),\left.\quad u\right|_{\partial \Omega}=0,
$$

where $t \in[0,1]$, so that when $t=1$ we have our original problem and when $t=0$ we have a problem of the type studied in Theorem 1 . We shall prove that the set of all possible solutions of (3.20) is bounded in $C^{1}(\bar{\Omega})$ independently of $t \in[0,1)$. Here the operator $E$ is defined by

$$
E u=L u+\lambda_{1} u+d_{1} u .
$$

Let us assume that the relation (3.6) does not hold true for solutions of (3.20), then there exist $\left(t_{n}\right)$ in the open interval $(0,1)$ and $\left(u_{n}\right)$ in $W^{2, p}(\Omega)$ with $\left|u_{n}\right|_{C^{1}} \geq n$ for each $n \in \mathbf{N}$ such that

$$
E u_{n}=t_{n}\left[d_{1} u_{n}-g\left(x, u_{n}\right)+h(x)\right],\left.\quad u_{n}\right|_{\partial \Omega}=0 .
$$

By proceeding as in the proof of Theorem 1 with $v_{n}=\left(u_{n} /\left|u_{n}\right|_{C^{1}}\right)$, we have that there exist $v \in W^{2, p}(\Omega), t \in(0,1]$ such that relations $(3.10)$ are fulfilled and $v$ solves the equation

$$
L v+\lambda_{1} v+\left[(1-t) d_{1}+t k_{v}^{+}(x)\right] v^{+}-\left[(1-t) d_{1}+t k_{v}^{-}(x)\right] v^{-}=0,\left.\quad v\right|_{\partial \Omega}=0
$$


where, by reasonings similar to those used in the proof of Theorem 1, for a.e. $x \in \Omega$

$$
\begin{gathered}
0 \leq(1-t) d_{1}+t k_{v}^{+}(x) \leq(1-t) d_{1}+t \Gamma_{+}(x) \leq d, \\
0 \leq(1-t) d_{1}+t k_{v}^{-}(x) \leq(1-t) d_{1}+t \Gamma_{-}(x) \leq \Gamma_{-}(x) .
\end{gathered}
$$

Hence, by Lemma 2 in which

$$
\begin{aligned}
& p_{+}(x)=(1-t) d_{1}+t k_{v}^{+}(x), \\
& p_{-}(x)=(1-t) d_{1}+t k_{v}^{-}(x),
\end{aligned}
$$

we deduce that (since $|v|_{C^{\prime}}=1$ ) either $v>0$ in $\Omega$ and $\partial v / \eta<0$ on $\partial \Omega$, or $v<0$ in $\Omega$ and $\partial v / \partial \eta>0$ on $\partial \Omega$. One can, now, reach a contradiction as in the proof of Theorem 1, with relation (3.17) replaced by

$$
L u_{n}+\lambda_{1} u_{n}+\left(1-t_{n}\right) d_{1} u_{n}+t_{n} g\left(x, u_{n}\right)=t_{n} h(x),\left.\quad u_{n}\right|_{\partial \Omega}=0,
$$

for each $n>n_{0}$. The proof is complete.

Remark 3. The sign condition (3.2) cannot be relaxed, as illustrated by a counterexample in [12], to hold only for all $u$ in $\mathbf{R}$ with $|u|>R>0$. However in case $\liminf _{u \rightarrow \infty} g(x, u)=g_{\infty}(x)$ is such that $\int g_{\infty}(x) \phi d x>0$, one may replace, in Theorem 2, the orthogonality condition (3.5) by a Landesman-Lazer condition

$$
\int h \phi d x<\int g_{\infty}(x) \phi d x
$$

and relaxing a little bit the sign condition (3.2). Therefore, our Theorem 2, with these obvious modifications, may be considered as an improvement of Theorem 4 in Berestycki and de Figueiredo $\left[4\right.$, p. 113] to the case when $\Gamma_{+}(x)$ is not necessarily identically zero. On the other hand, if $\Gamma_{-}$is a constant, then in the one dimensional case, one can relate explicitly $d$ to $\Gamma_{-}$by using the "shooting method". We refer to Drabek [13] and references therein. Note that the "shooting method" works only for the one-dimensional case, i.e. ordinary differential equations. If the Landesman-Lazer condition is not fulfilled our result of Theorem 2 is completely new in case of ordinary and partial differential equations.

The following result follows from a slight modification of the proof of Theorem 2 .

Theorem 3. Let us assume that the inequality (3.2) holds true. Moreover, suppose that the functions $\Gamma_{ \pm}(x)$, given in relation (3.1), are such that

$$
\Gamma_{+}(x)=0 \leq \Gamma_{-}(x)
$$

for a.e. $x \in \Omega$. Then the conclusion of Theorem 2 holds true. One can obtain a similar result when the roles $\Gamma_{+}$and $\Gamma_{-}$are interchanged.

Proof. We consider the following homotopy

$$
L u+\lambda_{1} u+(1-t) g_{1}(x, u)+t g(x, u)=t h(x),\left.\quad u\right|_{\partial \Omega}=0,
$$


where $g_{1}: \Omega \times \mathbb{R} \rightarrow \mathbb{R}$ is defined by

$$
g_{1}(x, u)= \begin{cases}(\alpha / 2) u & \text { for } u<0, \\ \ln (1+u) & \text { for } u \geq 0,\end{cases}
$$

and as before $t \in[0,1]$, so that when $t=1$ we have our original problem, and when $t=0$ we have a problem of the type studied in Theorem 1 .

As in the proof of Theorem 2, it suffices to show that the set of all possible solutions of (3.27) is bounded in $C^{1}(\bar{\Omega})$ independently of the parameter $t \in$ $[0,1)$. Note that for $t=0$ the set of all possible solutions of (3.27) is bounded in $C^{1}(\bar{\Omega})$ since $\lim _{u \rightarrow-\infty} g_{1}(x, u)=-\infty$ and $\lim _{u \rightarrow \infty} g_{1}(x, u)=\infty$ (see e.g. $[2,4,20])$.

Let us assume that the relation (3.6) does not hold true for solutions of (3.27), then there exist sequences $\left(t_{n}\right)$ in the open interval $(0,1)$ and $\left(u_{n}\right)$ in $W^{2, p}(\Omega)$ with $\left|u_{n}\right|_{C^{1}} \geq n$ for each $n \in \mathbb{N}$ such that

$$
L u_{n}+\lambda_{1} u_{n}+\left(1-t_{n}\right) g_{1}\left(x, u_{n}\right)+t_{n} g\left(x, u_{n}\right)=t_{n} h,\left.\quad u_{n}\right|_{\partial \Omega}=0 .
$$

Now, proceeding as in the proof of Theorem 2 with $v_{n}=u_{n} /\left|u_{n}\right|_{C^{1}}$, we can find $t \in(0,1]$ and $v \in W^{2, p}(\Omega)$ with $|v|_{C^{1}}=1, v_{n} \rightarrow v$ in $C^{1}(\bar{\Omega}), t_{n} \rightarrow t$ and $v$ solves the equation

$$
L v+\lambda_{1} v-\left[(1-t)(\alpha / 2)+t k_{v}^{-}(x)\right] v^{-}=0,\left.\quad v\right|_{\partial \Omega}=0,
$$

with

$$
0 \leq k_{v}^{-}(x) \leq \Gamma_{-}(x) \text { for a.e. } x \in \Omega .
$$

Taking the inner product (in $L^{2}(\Omega)$ ) of (3.30) with the eigenfunction $\phi$, we deduce that

$$
\int_{\Omega}\left[(1-t)(\alpha / 2)+t k_{v}^{-}\right] v^{-} \phi d x=0
$$

which implies that

$$
(1-t)(\alpha / 2)+t k_{v}^{-}(x)=0 \quad \text { whenever } v(x)<0,
$$

so that $v \in W^{2, p}(\Omega)$ solves the equation

$$
L v+\lambda_{1} v=0,\left.\quad v\right|_{\partial \Omega}=0,
$$

and therefore $v \in N\left(L+\lambda_{1} I\right) \backslash\{0\}$. one can reach a contradiction as in the proof of Theorem 2 and the proof is complete.

Let us give an example of a nonlinearity which is unbounded and such that neither monotonicity nor Landesman-Lazer condition is fulfilled.

Example. Let $g: \Omega \times \mathbf{R}$ be defined by

$$
g(x, u)= \begin{cases}c(x) u \sin ^{2} u & \text { for } x \in \Omega \text { and all } u \geq 0, \\ \Gamma_{-}(x) u \sin ^{2} u & \text { for } x \in \Omega \text { and all } u \leq 0\end{cases}
$$

where $\Gamma_{-}(x) \geq \lambda_{2}-\lambda_{1}, \Gamma_{-}$is an $L^{p}(\Omega)$-function; $c(x)=0$ on a subset $\Omega_{1}$ of $\Omega$ of positive measure and $0 \leq c(x) \leq d$, where $d=d\left(\Gamma_{-}\right)$is a positive 
constant associated to $\Gamma_{-}$by Lemma 2. (Note that $c(x)$ may be zero a.e. on $\Omega$.)

Obviously $g(x, u)$ is not monotone in its second variable and

$$
\limsup _{u \rightarrow-\infty} g(x, u)=0=\liminf _{u \rightarrow \infty} g(x, u) \text { for a.e. } x \in \Omega \text {. }
$$

On the other hand limsup $\sup _{u \rightarrow-\infty} g(x, u) / u=\Gamma_{-}(x)$ may cross any number of eigenvalues.

\section{NONSELFADJOINT SEMILINEAR RESONANCE PROBLEMS}

In this section, we shall state results similar to our Theorems 1 and 2 in case one is dealing with nonselfadjoint boundary value problems (1.2). It appears that the main tools for proving these results are Lemmas 3 and 4 herein.

Theorem 4. Let us assume that the inequality (3.2) holds true, and let $d^{*}>0$ be a constant given by Lemma 3.

Moreover, suppose that the functions $\Gamma_{+}, \Gamma_{-}$given in relation (3.1) are such that

$$
0 \leq \Gamma_{+}(x) \leq d^{*}, \quad 0 \leq \Gamma_{-}(x) \leq d^{*}
$$

for a.e. $x \in \Omega$. Then (1.2) has at least one solution $u \in W^{2, p}(\Omega) \cap H_{0}^{1}(\Omega)$ for any $h \in L^{p}(\Omega), p>N$, satisfying the orthogonality condition

$$
\int_{\Omega} h \phi^{*} d x=0
$$

(Obviously $u \in C^{1, \mu}(\bar{\Omega})$.)

Proof. We follow step by step the proof of Theorem 1 (up to the relation (3.17)), in which we replace the operator $L$ by the nonselfadjoint operator $A$ and we make use of Lemma 3 instead of Lemma 2.

Now, by taking the inner product (in $L^{2}(\Omega)$ ) of (3.17) by $\phi^{*}$ (replacing $L$ by $A$ ) and taking into account assumption (4.2), we deduce that

$$
\int_{\Omega} g\left(x, u_{n}\right) \phi^{*} d x<0
$$

for all $n>n_{0}$, so that we reach a contradiction as in the proof of Theorem 1, and the proof is complete.

Theorem 5. Let us assume that the inequality (3.2) holds true. Let $\Gamma_{-}(x) \geq c>$ $0, c \in \mathbb{R}$, a.e. on $\Omega, \Gamma_{-}$given in relation (3.1), and let $d=d\left(\Gamma_{-}\right)>0$ be a constant associated to $\bar{\Gamma}_{-}$by Lemma 4 . Moreover suppose that the function $\Gamma_{+}$, given in relation (3.1), is such that

$$
0 \leq \Gamma_{+}(x) \leq d
$$

for a.e. $x \in \Omega$. Then the conclusion of Theorem 4 holds true. 
One can obtain a similar result when the roles of $\Gamma_{+}$and $\Gamma_{-}$are interchanged. Proof. It is similar to the proof of Theorem 2 in which we introduce modifications similar to those in the proof of Theorem 4. The proof is complete.

One can also prove a result similar to Theorem 3 herein.

Acknowledgement. The authors wish to thank the referee for his careful reading of the manuscript and useful suggestions.

\section{REFERENCES}

1. R. A. Adams, Sobolev spaces, Academic Press, New York, 1975.

2. S. Ahmad, Nonselfadjoint resonance problems with unbounded perturbations, Nonlinear Anal., TMA 10 (1986), 147-156.

3. H. Amann and M. G. Crandall, On some existence theorems for semilinear elliptic equations, Indiana Univ. Math J. 27 (1978), 779-790.

4. H. Berestycki and D. G. de Figueiredo, Double resonance in semilinear elliptic problems, Comm. Partial Differential Eq. 6 (1981), 91-120.

5. J. M. Bony, Principe du maximum dans les espaces de Sobolev, C. R. Acad. Sci. Paris Ser. A 265 (1967), 333-336.

6. H. Brézis, Analyse fonctionnelle, théorie et applications, Masson, Paris, 1983.

7. H. Brézis and L. Nirenberg, Characterization of the ranges of some nonlinear operators and application to boundary value problems, Ann. Scuola Norm. Sup. Pisa 5 (1978), 225-326.

8. L. Cesari and R. Kannan, Qualitative study of a class of non-linear boundary value problems at resonance, J. Differential Equations 56 (1985), 63-81.

9. L. Cesari and P. Pucci, Existence theorems for nonselfadjoint semilinear elliptic boundary value problems, Nonlinear Anal., TMA 9 (1985), 1227-1241.

10. E. N. Dancer, On the Dirichlet problem for weakly nonlinear partial differential equations, Proc. Royal Soc. Edinburgh 76 (1977), 283-300.

11. D. G. de Figueiredo and J. P. Gossez, Conditions de non-résonance pour certains problèmes elliptiques semi-linéaires, C. R. Acad. Sci. Paris Sér. 1302 (1986), 543-545.

12. D. G. de Figueiredo and W. M. Ni, Perturbations of second order linear elliptic problems by nonlinearities without Landesman-Lazer condition, Nonlinear Anal., TMA 3 (1979), 629-634.

13. P. Drabek, On the resonance problem with nonlinearity which has arbitrary linear growth, J. Math. Anal. Appl. 127 (1987), 435-442.

14. S. Fucik, Surjectivity of operators involving linear noninvertible part and nonlinear compact perturbation, Funkcial. Ekvaci. 17 (1974), 73-83.

15. D. Gilbarg and N. S. Trudinger, Elliptic partial differential equations of second order, 2nd ed., Springer, New York, 1983.

16. J. V. A. Gonçalves, On bounded nonlinear perturbations of an elliptic equation at resonance, Nonlinear Anal., TMA 5 (1981), 57-60.

17. C. P. Gupta, Perturbations of second order linear elliptic problems by unbounded nonlinearities, Nonlinear Anal., TMA 6 (1982), 919-933.

18. C. P. Gupta, Solvability of a boundary value problem with the nonlinearity satisfying a sign condition, J. Math. Anal. Appl. 129 (1988), 482-492.

19. R. Iannacci and M. N. Nkashama, Unbounded perturbations of forced second order ordinary differential equations at resonance, J. Differential Equations 69 (1987), 289-309.

20. R. Iannacci and M. N. Nkashama, Nonlinear boundary value problems at resonance, Nonlinear Anal., TMA 11 (1987), 455-473.

21. R. Iannacci and M. N. Nkashama, Nonlinear two-point boundary value problems at resonance without Landesman-Lazer condition, Proc. Amer. Math. Soc. (in press). 
22. R. Kannan, J. J. Nieto and M. B. Ray, A class of nonlinear boundary value problems without Landesman-Lazer condition, J. Math. Anal. Appl. 105 (1985), 1-11.

23. M. G. Krein and M. A. Rutman, Linear operators leaving invariant a cone in a Banach space, Amer. Math. Soc. Transl. (2) 10 (1950), 199-325.

24. J. Mawhin, Topological degree methods in nonlinear boundary value problems, CBMS Regional Conf. Series in Math., no. 40, Amer. Math. Soc., Providence, R.I., 1979.

25. J. Mawhin, Nonresonance conditions of nonuniform type in nonlinear boundary value problems, Dynamical Systems II, (Bednarek and Cesari, eds.), Academic Press, New York, 1982, pp. 255-275.

26. J. Mawhin, A Neumann boundary value problem with jumping monotone nonlinearity, Delft Progress Report 10 (1985), 44-52.

27. J. Mawhin, J. R. Ward and M. Willem, Necessary and sufficient conditions for the solvability of a nonlinear two-point boundary value problem, Proc. Amer. Math. Soc. 93 (1985), 667-674.

28. J. Mawhin, J. R.Ward and M. Willem, Variational methods and semi-linear elliptic equations, Arch. Rational Mech. Anal. 95 (1986), 269-277.

29. J. Mawhin and M. Willem, Critical points of convex perturbations of some indefinite quadratic forms and semi-linear boundary value problems at resonance, Ann. Inst. Henri Poincaré 3 (1986), 431-453.

30. M. H. Protter and H. F. Weinberger, Maximum principles in differential equations, Springer, New York, 1984.

31. R. Schaaf and K. Schmitt, A class of nonlinear Sturm-Liouville problems with infinitely many solutions, Trans. Amer. Math. Soc. 306 (1988), 853-859.

32. M. Schechter, J. Shapiro and M. Snow, Solutions of the nonlinear problem $A u=N u$ in $a$ Banach space, Trans. Amer. Math. Soc. 241 (1978), 69-78.

33. J. R. Ward, Jr., A note on the Dirichlet problem for some semi-linear elliptic equations, (preprint 1986).

Dipartimento di Metodi e Modelli Matematici per le Scienze Applicate, Università "La Sapienza", Via A. Scarpa, 10, 00161 Roma, Italy

Department of Mathematical Sciences, Memphis State University, Memphis, TennesSEE 38152

Department of Mathematics, University of Alabama, Tuscaloosa, Alabama 35487 